Applied Mathematical Sciences, Vol. 14, 2020, no. 3, 133 - 140

HIKARI Ltd, www.m-hikari.com

https://doi.org/10.12988/ams.2020.912171

\title{
Current-Value Hamiltonian for an Age-Structured Optimal Control Problem
}

\author{
Alessandra Buratto, Luca Grosset *\%, and Elena Sartori \\ Dipartimento di Matematica "Tullio Levi-Civita" \\ Università degli Studi di Padova
}

This article is distributed under the Creative Commons by-nc-nd Attribution License. Copyright (c) 2020 Hikari Ltd.

\begin{abstract}
In this paper, we prove a set of sufficient optimality conditions with a current-value Hamiltonian for a family of age-structured optimal control problems with a discount factor in the objective functional.
\end{abstract}

Mathematics Subject Classification: 49K20, 49N90, 91B55

Keywords: Age-structured dynamics, Current-value Hamiltonian

\section{Introduction}

Many dynamic economic models require a discount factor in the objective functional, especially when performing a long term analysis. In this context, the current-value Hamiltonian approach has been introduced to analyze the optimality sufficient conditions for a classic optimal control problem, see e.g. [3], p. 47] and [13, p.99].

Recently, age-structured optimal control problems have been used to formalize and solve many problems. Without any claim to completeness, we can cite some applications to drug initiation [2], vintage capital [5], dynamic advertising [7], differential games [8, and immigration policy [14]. Necessary conditions for age-structured optimal control problems have been introduced in the seminal paper [4. This work represents the point of reference for a huge

\footnotetext{
${ }^{*}$ Corresponding author
} 
number of papers in which such necessary conditions are applied to analyze different models. A new approach to optimality conditions for an infinite-horizon age-structured optimal control problem is introduced in [15]. For what sufficiency is concerned, Mangasarian-type sufficient conditions for age-structured optimal control problems are presented in [11, and Arrow-type sufficient conditions are described in [10, where the author deals with infinite-horizon too.

Nevertheless, none of the cited papers use current-value Hamiltonian in their analysis. Even if this technique is standard for infinite-horizon optimal control problems (see e.g. [1, p. 285]), to the best of our knowledge, an explicit definition of current-value Hamiltonian for the age-structured optimal control problem has not been formalized yet.

In this paper, we fill this gap by introducing the current-value Hamiltonian into sufficient conditions for a simple age-structured optimal control problem. In our personal opinion, this approach can be useful for scientists dealing with age-structured optimal control problems, in particular to the ones devoted to Economics and Social Sciences, where problems may require non-trivial formulations. In such contexts, simplified sufficient conditions turn out to be essential to find the optimal solution.

The rest of the paper is organized as follows: In Section 2 we introduce the simple age-structured optimal control problem we want to analyze. In Section 3 we introduce the sufficient conditions which are based on the current-value Hamiltonian. Section 4 concludes with some suggestions for further research.

\section{An age-structured optimal control problem}

In this section, we present the age-structured optimal control problem we want to deal with. Perhaps, this is the simplest form for an age-structured optimal control problem. Its analysis is formally similar to the study of a standard infinite-horizon optimal control problem; hence this approach can be useful also for scientists coming from Economics or Social Sciences who manage very well this theory. The evolution of the state variable $y(t, a)$ is described by the following partial differential equation, where $(t, a) \in[0, \infty) \times[0, \omega]$,

$$
\partial_{t} y(t, a)+\partial_{a} y(t, a)=f(a, u(t, a), y(t, a)) .
$$

The initial condition for all $a \in[0, \omega]$, is

$$
y(0, a)=\alpha(a)
$$

and the boundary condition for all $t \in(0,+\infty)$ is

$$
y(t, 0)=\beta(t)
$$


Remark 2.1. We assume that $f \in C^{1}\left([0, \omega] \times \mathbb{R}^{2} ; \mathbb{R}\right), \alpha \in C^{0}([0, \omega] ; \mathbb{R})$, and $\beta \in C^{0}([0,+\infty) ; \mathbb{R})$, therefore, for any control $u \in C^{1}([0,+\infty) \times[0, \omega] ; \mathbb{R})$, there exists a unique almost everywhere differentiabl $\phi^{\dagger}$ state function $y(t, a)$ defined in $[0,+\infty) \times[0, \omega]$ that satisfies (1), (2), and (3).

In the partial differential equation (1) the lines $a=a_{0}+t$ are inflow characteristics for all $a_{0} \in \mathbb{R}$, hence we can use the information $\alpha(a)$ on the segment $\{0\} \times[0, \omega]$ and the information $\beta(t)$ on the semi-axis $[0,+\infty) \times\{0\}$ to find the solution of (1) using the method of characteristics (see e.g. [12]).

We choose the control in order to maximize, considering the overtaking criterion, the objective functional

$$
J(u)=\int_{0}^{+\infty} \int_{0}^{\omega} e^{-\rho t} g(a, u(t, a), y(t, a)) d a d t
$$

where $g \in C^{1}\left([0, \omega] \times \mathbb{R}^{2} ; \mathbb{R}\right)$. To characterize an optimal solution of this agestructured optimal control problem, we introduce the optimality conditions for age-structured control systems proved in [4]. This problem can be solved also by a direct computation along the characteristics lines, as explained in [16] and in [9]; however, we prefer to use the optimality conditions for age-structured control systems because we want to keep in mind the analogy of this problem with the standard infinite-horizon optimal control problem. Moreover, this approach can be the starting point for more complicated models where the use of the optimality conditions for age-structured control systems is essential.

\section{Standard sufficient conditions}

In this section, we introduce the standard sufficient conditions for age-structured control systems. We refer to the Arrow-type sufficient conditions introduced in [10]. Once again, we emphasize that we aim to present a simple approach to a simple version of the infinite-horizon age-structured optimal control problem. The sufficient conditions described in the following can be extended to a more general problem formulation, nevertheless, we prefer to present the basic version just to stress the main features of this issue (see [10] and [11] for a complete discussion about sufficient conditions in age-structured control systems).

Let us introduce the Hamiltonian function

$$
H(t, a, u, y, p) \doteq e^{-\rho t} g(a, u, y)+p f(a, u, y)
$$

\footnotetext{
${ }^{\dagger}$ Except at the points of the line $a=t$ where the function $y$ may be discontinuous
} 
associated to the age-structured optimal control problem characterized by the objective functional (4), the motion equation (1), and the boundary conditions (2) , (3). This is the standard Hamiltonian function for this kind of problems: see e.g. [5], [6], and [10]. As usual, $p$ is the adjoint function associated with the state variable $y$.

Theorem 3.1. Let us assume that:

i) the following equation defines a continuously differentiable function

$$
u^{\#}[t, a, y, p] \doteq \arg \max _{w \in \mathbb{R}} H(t, a, w, y, p)
$$

ii) we can find a solution $\left(y^{*}(t, a), p^{*}(t, a)\right)$ of the following system of PDEs

$$
\left\{\begin{array}{l}
\partial_{t} y(t, a)+\partial_{a} y(t, a)=f\left(a, u^{\#}[t, a, y(t, a), p(t, a)], y(t, a)\right) \\
\partial_{t} p(t, a)+\partial_{a} p(t, a)=-\partial_{y} H\left(t, a, u^{\#}[t, a, y(t, a), p(t, a)], y(t, a), p(t, a)\right) \\
y(0, a)=\alpha(a), \quad y(t, 0)=\beta(t) \\
p(t, \omega)=0, \quad \lim _{t \mapsto+\infty} p(t, a)=0 \quad \forall a \in[0, \omega]
\end{array}\right.
$$

iii) for all state functions $y(t, a)$ there exists a time $\tau>0$ such that for all $t>\tau$ and for almost all $a \in[0, \omega]$

$$
p^{*}(t, a)\left(y(t, a)-y^{*}(t, a)\right) \geq 0
$$

iv) the following inequality is satisfied for all $t \in[0,+\infty), a \in[0, \omega]$

$$
\partial_{y y}^{2} H\left(t, a, u^{\#}\left[t, a, y, p^{*}(t, a)\right], y, p^{*}(t, a)\right) \leq 0
$$

then the function

$$
u^{*}(t, a) \doteq u^{\#}\left[t, a, y^{*}(t, a), p^{*}(t, a)\right]
$$

is an optimal control for the age-structured optimal control problem characterized by the objective functional (4), the motion equation (1), and the boundary conditions (2), (3).

Proof. This proposition is an instance of the more general result proved in [10].

Now we want to rewrite these sufficient conditions in an equivalent way, as done in [1] in order to obtain an autonomous system. For standard optimal control problems this approach is well-known and can be found in a lot of textbooks (see e.g. [6]); however, this result is not explicitly described anywhere for age-structured optimal control problems. 
Let us introduce the current-value Hamiltonian function

$$
H_{c}(a, u, y, q) \doteq g(a, u, y)+q f(a, u, y)
$$

associated with the age-structured optimal control problem formulated above, where $q$ is the adjoint function associated with the state variable $y$.

Theorem 3.2. Let us assume that:

i) the following equation defines a continuously differentiable function

$$
u_{c}^{\#}[a, y, q] \doteq \arg \max _{w \in \mathbb{R}} H_{c}(a, w, y, q)
$$

ii) we can find a solution $\left(y^{*}(t, a), q^{*}(t, a)\right)$ of the system of PDEs

$$
\begin{cases}\partial_{t} y(t, a)+\partial_{a} y(t, a)=f\left(a, u_{c}^{\#}[a, y(t, a), q(t, a)], y(t, a)\right) & \\ \partial_{t} q(t, a)+\partial_{a} q(t, a)=-\partial_{y} H_{c}\left(a, u_{c}^{\#}[a, y(t, a), q(t, a)], y(t, a), q(t, a)\right)+ & +\rho q(t, a) \\ y(0, a)=\alpha(a), & y(t, 0)=\beta(t) \\ q(t, \omega)=0, & \lim _{t \mapsto+\infty} e^{-\rho t} q(t, a)=0, \quad \forall a \in[0, \omega]\end{cases}
$$

iii) for all state functions $y(t, a)$ there exists a time $\tau>0$ such that for all $t>\tau$ and for almost all $a \in[0, \omega]$

$$
q^{*}(t, a)\left(y(t, a)-y^{*}(t, a)\right) \geq 0
$$

iv) the following inequality is satisfied for all $a, t$

$$
\partial_{y y}^{2} H_{c}\left(a, u_{c}^{\#}\left[a, y, q^{*}(t, a)\right], y, q^{*}(t, a)\right) \leq 0
$$

then the function

$$
u^{*}(t, a) \doteq u_{c}^{\#}\left[a, y^{*}(t, a), q^{*}(t, a)\right]
$$

is an optimal control for the age-structured optimal control problem characterized by the objective functional (4), the motion equation (1), and the boundary conditions (2), (3).

Proof. Let us assume that functions $q^{*}(t, a)$ and $y(t, a)$ satisfy the assumptions of Theorem 3.2; we want to prove that the functions $p^{*}(t, a)=e^{-\rho t} q^{*}(t, a)$ and $y(t, a)$ satisfy the hypotheses of Theorem 3.1. This position suggests the following change of variable

$$
p \doteq e^{-\rho t} q
$$

Now we want to analyse how this change of variable modifies the Hamiltonian functions. We notice that (5) and (7) are connected by the following equality 


$$
e^{-\rho t} H_{c}(a, u, y, q)=H\left(t, a, u, y, e^{-\rho t} q\right)=H(t, a, u, y, p) .
$$

Being $e^{-\rho t}>0$ and not depending on $u$, a maximum point of $H_{c}(a, u, y, q)$ w.r.t. $u$ is a maximum point w.r.t. $u$ of $e^{-\rho t} H_{c}(a, u, y, q)$ too, so that

$$
u_{c}^{\#}[a, y, q]=\arg \max _{u \in \mathbb{R}} H_{c}(a, u, y, q)=u^{\#}\left[t, a, y, e^{-\rho t} q\right] .
$$

Now let us assume that $\left(y^{*}(t, a), q^{*}(t, a)\right)$ is a solution of the system of PDEs (8). The boundary conditions in (6) are trivially satisfied because, from the condition $|q(t, a)|<M$ for all $t \in[0,+\infty)$ and for all $a \in[0, \omega]$, it follows that

$$
\lim _{t \mapsto+\infty} e^{-\rho t} q(t, a)=0 .
$$

Using (9), the solution of the motion equation

$$
\partial_{t} y^{*}(t, a)+\partial_{a} y^{*}(t, a)=f\left(a, u_{c}^{\#}\left[a, y^{*}(t, a), q^{*}(t, a)\right], y^{*}(t, a)\right)
$$

becomes

$$
\partial_{t} y^{*}(t, a)+\partial_{a} y^{*}(t, a)=f\left(a, u^{\#}\left[a, y^{*}(t, a), q^{*}(t, a) e^{-\rho t}\right], y^{*}(t, a)\right),
$$

hence if $y^{*}(t, a)$ and $p^{*}(t, a)=q^{*}(t, a) e^{-\rho t}$ satisfy (6), then we can conclude. Moreover, if we consider the adjoint equation in (8); we have that

$$
\begin{aligned}
& \left(\partial_{t}+\partial_{a}\right)\left(q^{*}(t, a) e^{-\rho t}\right)= \\
& =\partial_{t}\left(q^{*}(t, a)\right) e^{-\rho t}-\rho q^{*}(t, a) e^{-\rho t}+\partial_{a}\left(q^{*}(t, a)\right) e^{-\rho t} \\
& =e^{-\rho t}\left(\left(\partial_{t}+\partial_{a}\right)\left(q^{*}(t, a)\right)\right)-\rho q^{*}(t, a) e^{-\rho t} \\
& =-\partial_{y}\left(e^{-\rho t} H_{c}\left(a, u_{c}^{\#}\left[a, y^{*}(t, a), q^{*}(t, a)\right], y^{*}(t, a), q^{*}(t, a)\right)\right) \\
& =-\partial_{y}\left(H\left(t, a, u_{c}^{\#}\left[a, y^{*}(t, a), q^{*}(t, a)\right], y^{*}(t, a), q^{*}(t, a) e^{-\rho t}\right)\right) \\
& =-\partial_{y}\left(H\left(t, a, u^{\#}\left[a, y^{*}(t, a), q^{*}(t, a) e^{-\rho t}\right], y^{*}(t, a), q^{*}(t, a) e^{-\rho t}\right)\right)
\end{aligned}
$$

hence $y^{*}(t, a)$ and $p^{*}(t, a)=q^{*}(t, a) e^{-\rho t}$ satisfy (6).

Finally, if the function $H_{c}\left(a, u_{c}^{\#}\left[a, y, q^{*}(t, a)\right], y, q^{*}(t, a)\right)$ is concave in $y$, then the function $H\left(a, u^{\#}\left[a, y, q^{*}(t, a) e^{-\rho t}\right], y, q^{*}(t, a) e^{-\rho t}\right)$ is concave in $y$ too. In detail we have:

$$
\begin{aligned}
& \partial_{y y}^{2} H_{c}\left(a, u_{c}^{\#}\left[a, y, q^{*}(t, a)\right], y, q^{*}(t, a)\right) \leq 0 \\
& \partial_{y y}^{2}\left(e^{-\rho t} H_{c}\left(a, u_{c}^{\#}\left[a, y, q^{*}(t, a)\right], y, q^{*}(t, a)\right)\right) \leq 0 \\
& \partial_{y y}^{2}\left(H\left(a, u_{c}^{\#}\left[a, y, q^{*}(t, a)\right], y, q^{*}(t, a) e^{-\rho t}\right)\right) \leq 0 \\
& \partial_{y y}^{2}\left(H\left(a, u^{\#}\left[a, y, q^{*}(t, a) e^{-\rho t}\right], y, q^{*}(t, a) e^{-\rho t}\right)\right) \leq 0
\end{aligned}
$$

The result we have just proved is useful because it allows one to move from the non-autonomous system of PDEs (6), to the autonomous one (8). Now, under suitable hypotheses, we can find an equilibrium point for the system (8) and we can prove its stability.

A similar result can be shown for the catching up criterion and the sporadically catching up criterion too. 


\section{Conclusions}

In this paper, we have introduced the current-value Hamiltonian for a simple age-structured optimal control problem. Using the standard technique described for infinite-horizon optimal control, we defined the discount adjoint function and found sufficient conditions for this kind of problem as an autonomous system of PDEs. We restrict our analysis to a particular family of age-dependent optimal control problems, that turns out to be commonly used in Economics or Social Sciences applications. This approach permits to solve these types of problems without handling differential calculus in infinitedimensional spaces. The approach described in this paper can be the starting point for further applications. A promising research area in which this result can be applied is the theory of differential games, especially with applications to Economics and Social Sciences, where the equilibrium points play an essential role and many problems can be formulated with an age-dependent structure.

\section{References}

[1] Acemoglu D., Introduction to Modern Economic Growth, Princeton University Press, Princeton, 2003.

[2] Almeder C., Caulkins J.P., Feichtinger G., and Tragler G., An agestructured single-state drug initiation model-cycles of drug epidemics and optimal prevention programs, Socio-Economic Planning Sciences, 38 (1) (2004), 91-109. https://doi.org/10.1016/S0038-0121(03)00030-2

[3] Dockner E.J., Jorgensen S., Van Long N., and Sorger G., Differential Games in Economics and Management Science, Cambridge University Press, Cambridge, 2000.

[4] Feichtinger G., Tragler G., and Veliov V.M., Optimality conditions for agestructured control systems, Journal of Mathematical Analysis and Applications, 288 (1) (2003), 47-68. https://doi.org/10.1016/j.jmaa.2003.07.001

[5] Feichtinger G., Hartl R.F., Kort P.M., and Veliov V., Anticipation effects of technological progress on capital accumulation: a vintage capital approach, Journal of Economic Theory, 126 (1) (2006), 143-164. https://doi.org/10.1016/j.jet.2004.10.001

[6] Grass D., Caulkins J.P., Feichtinger G., Tragler G., and Behrens D.A., Optimal Control of Nonlinear Processes, Springer-Verlag, Heidelberg, 2008. https://doi.org/10.1007/978-3-540-77647-5 
[7] Grosset L. and Viscolani B., Advertising for the introduction of an agesensitive product, Optimal Control Applications and Methods, 26 (3) (2005), 157-167. https://doi.org/10.1002/oca.758

[8] Grosset L. and Viscolani B., Age-structured linear-state differential games, European Journal of Operational Research, 254 (1) (2016), 269-278. https://doi.org/10.1016/j.ejor.2016.03.025

[9] Krastev V., Optimal Dynamic Advertising Strategy Under Age-Specific Market Segmentation, AIP Conference Proceedings, 1410 (1) (2011), 296302. https://doi.org/10.1063/1.3664382

[10] Krastev V., Arrow-type sufficient conditions for optimality of agestructured control problem, Central European Journal of Mathematics, 11 (6) (2013), 1094-1111. doi:10.2478/s11533-013-0219-7

[11] Krastev V., Mangasarian-type sufficient optimality conditions for agestructured control problem with state constraints. An application to investment in vintage capital, Serdica Mathematical Journal, 39 (2) (2013), 155-188.

[12] Salsa S., Partial Differential Equations in Action, Springer, Milan, 2008. https://doi.org/10.1007/978-3-319-15093-

[13] Seierstad A. and Sydsaeter K., Optimal Control Theory with Economic Applications, North-Holland, Amsterdam, 1987.

[14] Simon C., Skritek B., and Veliov V., Optimal immigration age-patterns in populations of fixed size, Journal of Mathematical Analysis and Applications, 405 (1) (2013), 71-89. https://doi.org/10.1016/j.jmaa.2013.03.061

[15] Skritek B. and Veliov V.M., On the Infinite-Horizon Optimal Control of Age-Structured Systems, Journal of Optimization Theory and Applications, 167 (1) (2015), 243-271. https://doi.org/10.1007/s10957-014-0680-x

[16] Veliov V.M., Optimal control of heterogeneous systems: Basic theory, Journal of Mathematical Analysis and Applications, 346 (1) (2008), 227242. https://doi.org/10.1016/j.jmaa.2008.05.012

Received: January 31, 2020; Published: February 25, 2020 